\title{
VUV detection in large-area avalanche photodiodes as a function of temperature
}

\author{
J.A.M. Lopes ${ }^{a}$, L.M.P. Fernandes ${ }^{a}$, J.M.F. dos Santos ${ }^{a}$, , R.E. Morgado ${ }^{b}$, \\ C.A.N. Conde ${ }^{\mathrm{a}}$ \\ ${ }^{a}$ Departamento de Física, da Universidade de Coimbra, Coimbra 3004-516, Portugal \\ ${ }^{\mathrm{b}}$ Los Alamos National Laboratory, New Mexico, NM 87545, USA
}

\begin{abstract}
The response of a Peltier-cooled large-area avalanche photodiode to VUV-light is investigated as a function of the operation temperature. The reduction of the temperature down to $5^{\circ} \mathrm{C}$ will improve both photodiode light-level detection limit and statistical fluctuations; further temperature reduction will not result in improved performance. Optimum operation characteristics are already achieved for gains around 100 and do not depend significantly on the operation temperature. The relative variation of the gain with temperature increases with the biasing voltage presenting values that are almost a factor of two higher than for visible light detection.
\end{abstract}

(C) 2003 Elsevier Science B.V. All rights reserved.

PACS: $07.60 . \mathrm{Rd} ; 29.40 . \mathrm{Mc} ;$ 85.60.Dw

Keywords: LAAPD; VUV; Photon-detection

\section{Introduction}

Avalanche photodiodes (APDs) have increasingly assumed important roles in instrumentation for medium- and high-energy physics. Particularly, they present alternatives to UV- and visiblephoton detectors, as well as to X-ray detectors. They are compact, have low-power consumption and are simple operation devices. Operational characteristics as high quantum efficiency, relatively high internal gain and insensitiveness to strong magnetic fields, combined with relatively

\footnotetext{
*Corresponding author. Tel.: + 351-239-410667; fax: + 351239-829158.

E-mail address: jmf@gian.fis.uc.pt (J.M.F.dos Santos).
}

low response to ionising particles, make them competitive for using in the electromagnetic calorimeter of the CMS detector at the LHC $[1,2]$. Also, their application to PET devices is under investigation $[3,4]$. Recently, their application as the VUV photosensor in gas proportional scintillation counters (GPSC) has been investigated $[5,6]$. It was shown that VUV large area APDs (LAAPDs) can replace the photomultiplier tubes with advantages.

The dependence of photodiode gain on the temperature requires temperature stabilisation and/or compensation during measurements, which is a drawback for many applications. In particular, the knowledge of the gain variation with the temperature is important to allow for corrections 
due to small drifts in the operation temperature. This variation has been studied for visible-light detection reaching values about $3 \% /{ }^{\circ} \mathrm{C}$ for maximum gains [7]. However, it has been proved that some LAAPD characteristics are different for visible- and VUV-light detection, namely linearity effects [8] and the response under intense magnetic fields [9]. While visible photons interact deeper in the diode, VUV photons interact within the first few atomic layers: penetration depths in $\mathrm{Si}$ are about $5 \mathrm{~nm}$ and $1 \mu \mathrm{m}$ for 172- and 520-nm photons [10], respectively.

In the present work we will use a Peltier-cooled LAAPD [7] as the VUV scintillation readout of a xenon-GPSC to study the photodiode response to VUV as a function of the temperature. The photodiode gain and overall statistical fluctuations will be studied for different diode temperatures.

\section{Description}

The LAAPD is used as the VUV photosensor of a GPSC. Through the last decades GPSCs have been studied in detail and its use was mainly directed to X-ray spectrometry. However, the electroluminescence mechanism characteristic of the GPSC operation can be used for VUV production with known photon output (e.g. $[5,6])$.

The GPSC integrated with the LAAPD is depicted schematically in Fig. 1. It features a 3.1$\mathrm{cm}$ deep absorption region, a $0.9-\mathrm{cm}$ scintillation region, and is filled with pure xenon at 825 Torr $\left(\sim 1.09 \times 10^{5} \mathrm{~Pa}\right)$ continuously purified by a gettering device. G1 and G2 are highly transparent stainless-steel wire mesh grids used to establish the electric fields inside the detector. The LAAPD has a 16-mm active diameter and presents $105 \%$ quantum efficiency at $172 \mathrm{~nm}$ [7].

To produce a fixed amount of VUV photons incident on the LAAPD, 5.9-keV X-rays $\mathrm{Mn} \mathrm{K}_{\alpha}$ $\mathrm{X}$-rays from a ${ }^{55} \mathrm{Fe}$ radioactive source, with the $\mathrm{K}_{\alpha}$-line absorbed by a chromium foil, were allowed to interact in the GPSC. The 5.9-keV Xrays interact in the drift region producing a cloud of $N_{\mathrm{e}}=E_{x} / w=269$ primary electrons, where $E_{x}=5900 \mathrm{eV}$ and $w \sim 22 \mathrm{eV} /$ ion pair. The primary

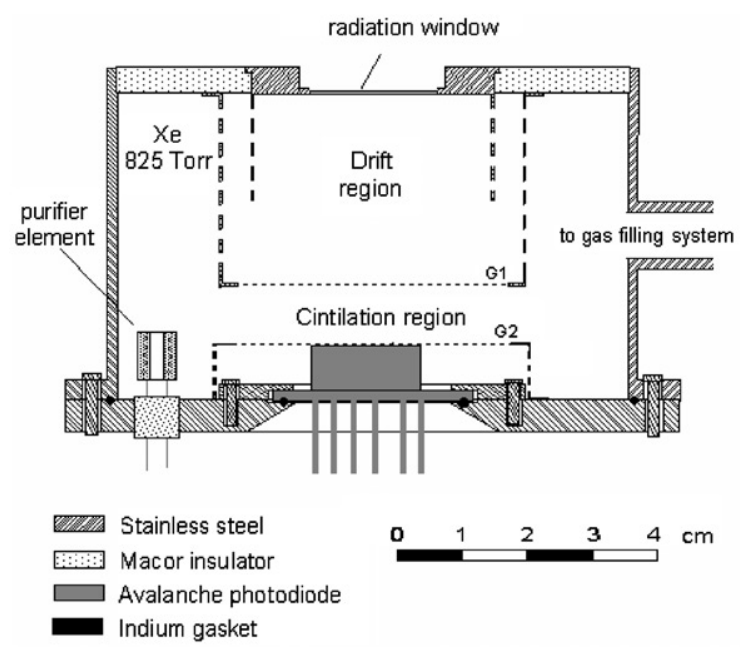

Fig. 1. Schematic of the GPSC with the Peltier-cooled LAAPD photosensor.

electron cloud drifts towards the scintillation region, under the influence of a weak electric field between the entrance window and grid G1, chosen to be below the xenon scintillation threshold. In the scintillation region, the electrons gain energy in the high electric field between grids G1 and G2 that is chosen to be above the scintillation threshold but below the electron impact ionisation threshold. The radiation window is biased at negative high voltage and electric fields of 0.8 and $6.2 \mathrm{~V} \mathrm{~cm}^{-1}$ Torr $^{-1}$ were used in the drift and scintillation regions, respectively.

For the described conditions about 118 VUV photons were collected in the LAAPD for each primary electron traversing the scintillation region [5], resulting in a total of about $3.2 \times 10^{4}$ detected photons per X-ray interaction. The scintillation photons incident on the LAAPD produced photoelectrons that were amplified by the avalanche process. The signals were fed through a Canberra 2004 preamplifier and a HP5582A amplifier, with $2-\mu$ s shaping time constants, to a Nucleus PCA-II MCA. The 5.9-keV X-ray pulse-height distributions were fitted to a gaussian function superimposed on a linear background, from which the centroid and the full-width at half-maximum were determined. 


\section{Experimental results and discussion}

In Fig. 2 we depict the LAAPD gain as a function of the biasing voltage for different LAAPD operation temperatures. The gain increases with decreasing temperature, with maximum achievable gains increasing from 300 to values above 700 as the temperature decreases from $25^{\circ} \mathrm{C}$ to $-5^{\circ} \mathrm{C}$. For each measurement, the diode temperature was stabilised within $\pm 0.1^{\circ} \mathrm{C}$.

From the data of Fig. 2 we obtained the LAAPD gain as a function of the temperature, for different biasing voltages, Fig. 3. For each voltage, the gain relative variation is almost constant through the measured temperature range, increasing from $2.7 \%$ to $5.6 \% /{ }^{\circ} \mathrm{C}$ as the LAAPD bias increases from 1633 to $1826 \mathrm{~V}$. For gains around 200 and for room temperature, the results

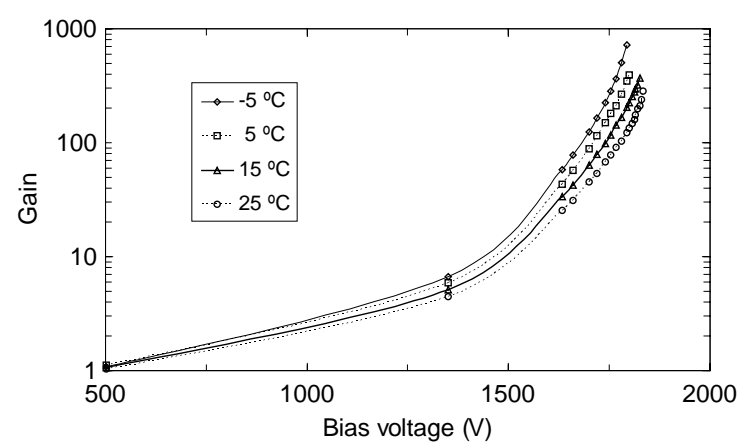

Fig. 2. LAAPD gain as a function of the biasing voltage for different operation temperatures.

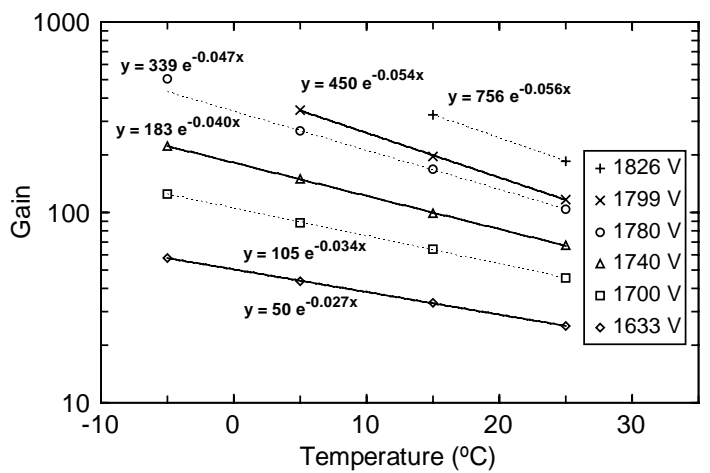

Fig. 3. LAAPD gain as a function of the operation temperature for different biasing voltages. show relative amplitude variations that are almost a factor of two higher than the $\sim 3 \% /{ }^{\circ} \mathrm{C}$ obtained for visible light [7].

The electronic noise-tail limits the minimum number of detectable VUV-photons (MDP), defined as the number of photons corresponding to the amplitude-limit where the electronic-noise tail rises above the background level. This value is a measure of how effective the LAAPD gain is in separating a VUV-light signal from the noise. Fig. 4 depicts the MDP as a function of the gain for the different operation temperatures. The MDP stabilises for gains around 100 and improves with decreasing LAAPD temperature but, below $5^{\circ} \mathrm{C}$, the observed improvement is not significant.

The statistical fluctuations associated with the light amplification process in GPSCs are negligible compared to those associated with the primary electron cloud formation and with the photon detection and signal amplification in the photosensor. The statistical fluctuations associated with the primary electron cloud formation in a gaseous detector are well known [11] and, thus, we can estimate the statistical fluctuations associated with the LAAPD VUV detection from the measured statistical fluctuations observed for the 5.9-keV Xrays in the GPSC. Fig. 5 presents the relative fluctuations estimated for the LAAPD detection of VUV photons as a function of the diode gain, for different diode temperatures. The correlation between Figs. 4 and 5 is noticeable: for each temperature, the minimum energy resolution is

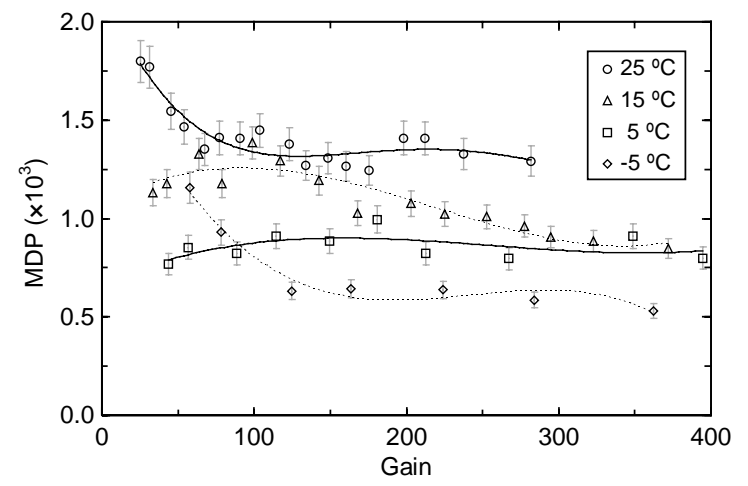

Fig. 4. Minimum number of detectable VUV photons (MDP) in the LAAPD as a function of the gain for different operation temperatures. 


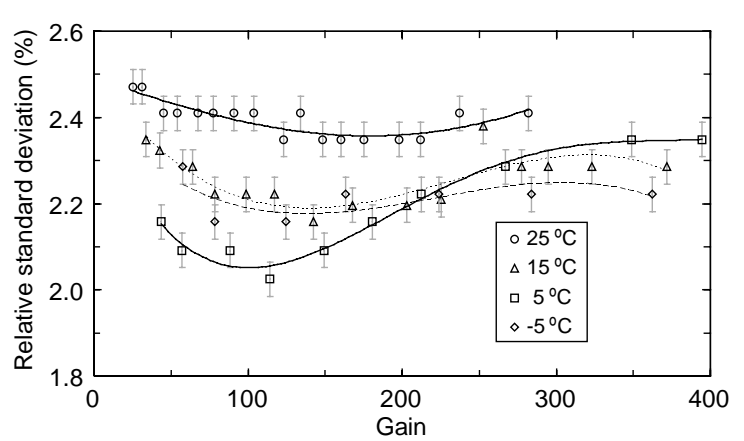

Fig. 5. Relative standard deviation for the fluctuations associated with the detection of $3.2 \times 10^{4}$ VUV-photons as a function of the LAAPD gain, for different operation temperatures.

achieved for the lowest gains where the MDP stabilises, and operation temperatures below $5^{\circ} \mathrm{C}$ will not result in a significant improvement of the statistical fluctuations.

\section{Conclusions}

The VUV photon detection with LAAPD improves with decreasing photodiode operation temperature. However, for temperatures below $5^{\circ} \mathrm{C}$ the improvement is not significant. Optimum operation characteristics are already achieved for gains around 100 and do not depend significantly on the operation temperature. The significant increase of the excess noise factor [12] (a factor that is related to the gain fluctuations in the multiplication process) for high gains becomes dominant, resulting in degraded performance of the photodiode.

The relative variation of gain with temperature increases with the biasing voltage, presenting values that increase from $2.7 \% /{ }^{\circ} \mathrm{C}$ to $5.6 \% /{ }^{\circ} \mathrm{C}$ when the voltage is increased from 1633 to $1826 \mathrm{~V}$. These values are almost a factor of two higher than for visible light detection.

\section{Acknowledgements}

This work was performed in Unit 217/94, FCTUC. Support is acknowledged to project CERN/FIS/43785/01. LMP Fernandes acknowledges grant SFRH/BD/5426/2001 from Fundação para a Ciência e a Tecnologia, Portugal.

\section{References}

[1] A. Karar, Y. Musienko, J.Ch. Vanel, Nucl. Instr. and Meth. A 428 (1999) 413.

[2] K. Deiters, Y. Musienko, S. Nicol, et al., Nucl. Instr. and Meth. A 442 (2000) 193.

[3] A. Ruru Chen, A. Fremout, S. Tavernier, et al., Nucl. Instr. and Meth. A 433 (1999) 637.

[4] V.N. Solovov, V. Chepel, M.I. Lopes, et al., IEEE Trans. Nucl. Sci. NS-47 (2000) 1307.

[5] J.A.M. Lopes, J.M.F. Dos Santos, R.E. Morgado, C.A.N. Conde, IEEE Trans. Nucl. Sci. NS-48 (2001) 312.

[6] C.M.B. Monteiro, J.A.M. Lopes, P.C.P.S. Simões, et al., IEEE Trans. Nucl. Sci. NS-48 (2001) 1081.

[7] Advanced Photonix, Camarillo, CA, USA: Windowless large area APDs $(5 \times \phi$ deep-UV series $)$, Application Notes.

[8] L.M.P. Fernandes, J.A.M. Lopes, C.M.B. Monteiro, et al., Nucl. Instr. and Meth. A 478 (2002) 395.

[9] L.M.P. Fernandes, A. Antognini, M. Boucher, et al., Nucl. Instr. and Meth. A 498 (2003) 362.

[10] T.W. Barnad, MM.I. Crockett, J.C. Ivaldi, et al., Anal. Chem. 65 (1993) 1231.

[11] J.M.F. dos Santos, J.A.M. Lopes, J.F.C.A. Veloso, et al., X-ray Spectrom. 30 (2000) 373.

[12] M. Moszynski, M. Szawlowski, M. Kapusta, M. Balcerzyk, D. Wolski, Nucl. Instr. and Meth. A 442 (2000) 230. 